\title{
Variance-Constrained Distributed Filtering for Time-varying Systems with Multiplicative Noises and Deception Attacks over Sensor Networks
}

\author{
Lifeng Ma, Zidong Wang, Fellow, IEEE, Qing-Long Han, Senior Member and Hak-Keung Lam
}

\begin{abstract}
This paper is concerned with the varianceconstrained distributed filtering problem for a class of timevarying systems subject to multiplicative noises, unknown but bounded disturbances and deception attacks over sensor networks. The available measurements at each sensing node are collected not only from the individual sensor but also from its neighbors according to the given topology. A new deception attack model is proposed where the malicious signals are injected by the adversary into both control and measurement data during the process of information transmission via the communication network. By resorting to the recursive linear matrix inequality approach, a sufficient condition is established for the existence of the desired filter satisfying the prespecified requirements on the estimation error variance. Subsequently, an optimization problem is formulated in order to seek the filter parameters ensuring the locally optimal filtering performance at each time instant. Finally, an illustrative example is presented to demonstrate the effectiveness and applicability of the proposed algorithm.
\end{abstract}

Index Terms-Distributed filtering, multiplicative noises, deception attacks, variance constraints, sensor networks, unknown but bounded disturbances

\section{INTRODUCTION}

The rapid development of microelectronic technologies over the past few decades has boosted the utilization of networks which consist of a large number of devices implemented distributively for sensing, communication as well as actuating [28]. A typical example is the sensor network which has been found wide applications ranging from various industrial branches to critical infrastructures such as military facilities and power grids, see [3], [23] and the references therein. In particular, the state estimation or filtering problems over sensor

This work was supported in part by the Fundamental Research Funds for the Central Universities under Grant 30916011337, the National Natural Science Foundation of China under Grants 61304010, the Postdoctoral Science Foundation of China under Grant 2014M551598, International Postdoctoral Exchange Fellowship from the China Postdoctoral Council, and Alexander von Humboldt Foundation of Germany.

L. Ma is with the School of Automation, Nanjing University of Science and Technology, Nanjing 210094, China, and also with the Key Laboratory of Intelligent Perception and Systems for High-Dimensional Information of Ministry of Education (Nanjing University of Science and Technology), Nanjing, 210094, China. (Email: malifengenjust.edu.cn)

Z. Wang is with the Department of Computer Science, Brunel University London, Uxbridge, Middlesex, UB8 3PH, United Kingdom. (Email: Zidong. Wangebrunel.ac.uk)

Q.-L. Han is with the School of Software and Electrical Engineering, Swinburne University of Technology, John Street, Hawthorn, Melbourne, VIC 3122, Australia.

H.-K. Lam is with Department of Informatics, School of Natural \& Mathematical Science, King's College London, Strand Campus, WC2R 2LS, United Kingdom. networks have posed several emerging challenges, which have attracted an ever-increasing research attention within the signal processing and control community.

So far, considerable effort has been devoted to the investigation of the distributed filtering problems and a number of strategies have been developed based on the Kalman filtering theory or the $H_{\infty}$ filtering theory, see [5], [6], [13], [14], [16], [26], [31] for some recent results. As is well known, the Kalman filtering technique requires an assumption of Gaussian distributions for the process and measurement noises, while the $H_{\infty}$ theory can be utilized in the occasion when the disturbances are assumed to have bounded energy. However, in many real-world engineering practice, due to a variety of reasons (e.g., man-made electromagnetic interference), it is much more appropriate to model the disturbances/noises as signals that are unknown but bounded in certain sets rather than Gaussian noises or energy-bounded disturbances [8], [12], [33]. Obviously, in such a case, the aforementioned conventional techniques based on Kalman filtering or $H_{\infty}$ filtering frameworks are no longer effective. Consequently, the filtering problems for systems subject to the so-called unknown but bounded noises have exerted tremendous fascination on researchers as well as engineers within the signal processing community. So far, quite a few methodologies have been exploited, see e.g. [9], [24]. Nevertheless, in the general context of sensor networks, little progress has been made on the corresponding filtering problems owing probably to the difficulty in quantifying the filtering performance with respect to the unknown but bounded noises as well as the complexity which stems from the coupling between communication topology and the system dynamics.

Along with the pervasive utilization of open yet unprotected communication networks, the sensor networks are vulnerable to cyber threat [10]. As a result, the security of network, which is of utmost importance in the networked-related systems, has provoked an increasing research interest and a multitude of results have been reported in the literature, see [29] and the references therein. In general, there are mainly two types of cyber attacks which can affect the systems behavior directly or through feedback, namely, the denial-of-service (DoS) attacks [21] and the deception attacks [7]. Different from the DoS attack which deteriorates the system performance by preventing the information from reaching the destination, the deception attack aims at manipulating the system toward the adversaries' desired behaviors by injecting deception information to the control actions or system measurements. A quintessen- 
tial example of deception attack should be cited is that in the context of target tracking, the electronic countermeasure (ECM) techniques are always developed to deceive radars. By manipulating and rebroadcasting the Doppler information of the target, the deception signals can be injected and maintained through frequency-shifted copies of the radar's signals, thereby degrading the tracking performance [30].

To tackle the filtering/control problems for the systems under cyber attacks, several approaches have been developed, including linear programming [29], linear matrix inequality method [18], [27], game theory approach [38], to name but a few key ones. However, when it comes to the distributed filtering issues over sensor networks, the corresponding results have been scattered, although some interesting initial results have appeared, see e.g. [32], [34]. To the best of the authors' knowledge, up to now, the research on distributed filtering is far from adequate when the communication networks are affected by attacks. The difficulty probably lies in the lack of appropriate attack models which, on one hand, could comprehensively reflect the engineering practice, and on the other, can be handled systematically within the existing frameworks. There are still a number of open yet challenging problems deserving further investigation.

In response to the above discussion, it is our objective in this paper to design a distributed filter for the discrete time-varying systems with multiplicative noises, unknown but bounded disturbances and deception attacks such that the estimation error variance of each sensing node is constrained by a prespecified upper bound at each time instant. Note that the specific time-varying nature of the addressed system imposes substantial challenges on both performance analysis and filter design, not to mention the difficulties stemming from the coupling between the communication topology and the deception attacks, especially when the error variances are required to satisfy certain upper bounds at each time step. Therefore, we shall make the first of the few attempts to develop new paradigms to solve the so-called varianceconstrained distributed filtering problem subject to deception attacks over sensor networks.

The main contributions can be highlighted as follows: (i) a unified framework is established within which the varianceconstrained distributed filtering problem can be conveniently handled in the presence of multiplicative noises, unknown but bounded disturbances and deception attacks; (ii) the proposed deception attack model is novel, which provides a better way to reflect the engineering reality in a comprehensive way by simultaneous consideration of several network-induced complexities; and (iii) a sufficient condition is proposed to recursively determine the filter parameters capable of guaranteeing the prespecified upper bound on the estimation error variances at each time instant.

The rest of this paper is organized as follows: Section II formulates the variance-constrained distributed filter design problem for the discrete time-varying system subject to multiplicative noises, unknown but bounded disturbances and deception attacks. The main results are presented in Section III where a sufficient condition for the existence of the desired filter is given in terms of recursive linear matrix inequalities.
Section IV gives a numerical example. Section $\mathrm{V}$ is our conclusion.

Notation The notation used here is fairly standard except where otherwise stated. $\mathbb{R}^{n}$ denotes the $n$-dimensional Euclidean space. $\mathbf{1}_{n}$ denotes an $n$-dimensional column vector with all ones. $I_{n}$ denotes the identity matrix of $n$ dimensions. The notation $X \geq Y$ (respectively $X>Y$ ) where $X$ and $Y$ are symmetric matrices, means that $X-Y$ is positive semi-definite (respectively positive definite). The superscript " $\mathrm{T}$ " denotes the transpose. $Z^{+}$stands for all the positive integers. $\operatorname{diag}\left\{F_{1}, F_{2}, \ldots\right\}$ denotes a block diagonal matrix whose diagonal blocks are given by $F_{1}, F_{2} \ldots$ The notation $\operatorname{diag}_{n}\left\{A_{i}\right\}$ represents the block diagonal matrix $\operatorname{diag}\left\{A_{1}, A_{2}, \ldots, A_{n}\right\}$. The notation $\operatorname{col}_{n}\left\{x_{i}\right\}$ denotes the column vector $\left[\begin{array}{llll}x_{1}^{\mathrm{T}} & x_{2}^{\mathrm{T}} & \ldots & x_{n}^{\mathrm{T}}\end{array}\right]^{\mathrm{T}}$. For matrices $A \in \mathbb{R}^{m \times n}$ and $B \in \mathbb{R}^{p \times q}$, their Kronecker product is a matrix in $\mathbb{R}^{m p \times n q}$ denoted as $A \otimes B$. $\|a\|_{2}^{2}$ where $a$ is a vector represents $a^{\mathrm{T}} a$, while $\|a\|_{A}^{2}$ means $a^{\mathrm{T}} A a \cdot \operatorname{tr}[A]$ means the trace of matrix $A$.

\section{PROBlem Formulation}

In this paper, it is assumed that the sensor network has $N$ sensor nodes which are distributed in the space according to a specific interconnection topology characterized by a directed graph $\mathscr{G}=(\mathscr{V}, \mathscr{M}, \mathscr{L})$, where $\mathscr{V}=\{1,2, \ldots, N\}$ denotes the set of sensor nodes, $\mathscr{M} \subseteq \mathscr{V} \times \mathscr{V}$ is the set of edges, and $\mathscr{L}=$ $\left[\theta_{i j}\right]_{N \times N}$ is the nonnegative adjacency matrix associated with the edges of the graph, i.e., $\theta_{i j}>0 \Leftrightarrow$ edge $(i, j) \in \mathscr{M}$, which means that there is information transmission from sensor $j$ to sensor $i$. If $(i, j) \in \mathscr{M}$, then node $j$ is called one of the neighbors of node $i$. Also, we assume that $\theta_{i i}=1$ for all $i \in \mathscr{V}$, and therefore, $(i, i)$ can be regarded as an additional edge. The set of neighbors of node $i \in \mathscr{V}$ plus the node itself is denoted by $\mathscr{N}_{i} \triangleq\{j \in \mathscr{V} \mid(i, j) \in \mathscr{M}\}$.

\section{A. System model}

Consider a discrete time-varying system described by

$$
x_{k+1}=\left(A_{k}+\sum_{l=1}^{q} \alpha_{l, k} A_{l, k}\right) x_{k}+B_{k} u_{k}+D_{k} w_{k},
$$

with the measurements from $N$ sensors given by

$$
y_{i, k}=C_{i, k} x_{k}+E_{i, k} v_{k}, \quad i=1,2, \ldots, N
$$

where $x_{k} \in \mathbb{R}^{n}, u_{k} \in \mathbb{R}^{m}$ and $y_{i, k} \in \mathbb{R}^{p}$ are, respectively, the state, the known input and the measurement output of sensor $i ; \alpha_{l, k} \in \mathbb{R}(l=1,2, \ldots, q)$ are sequences of uncorrelated zero-mean Gaussian noises with unitary covariances; $w_{k} \in \mathbb{R}^{\omega}$ and $v_{k} \in \mathbb{R}^{\nu}$ represent the unknown but bounded process and measurement disturbances, respectively; $A_{k}, B_{k}, D_{k}, C_{i, k}$ and $E_{i, k}$ are real-valued time-varying matrices of compatible dimensions. The following definitions as well as assumptions are needed for the further development.

Definition 1: Let $\Psi_{1}$ and $\Psi_{2}$ be some real matrices with $\Psi \triangleq \Psi_{2}-\Psi_{1}>0$. A nonlinearity $\varphi(\cdot): \mathbb{R}^{n} \mapsto \mathbb{R}^{n}$ is said to satisfy the sector condition with respect to $\Psi_{1}$ and $\Psi_{2}$ if

$$
\left(\varphi(\varepsilon)-\Psi_{1} \varepsilon\right)^{\mathrm{T}}\left(\varphi(\varepsilon)-\Psi_{2} \varepsilon\right) \leq 0, \quad \forall \varepsilon \in \mathbb{R}^{n} .
$$




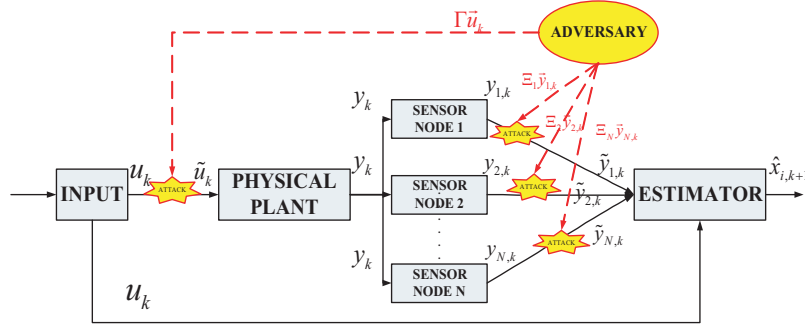

Fig. 1. Deception attack model.

In this case, the sector-bounded nonlinearity $\varphi(\cdot)$ is said to belong to the sector $\left[\Psi_{1}, \Psi_{2}\right]$.

Definition 2: A bounded ellipsoid $\mathscr{E}(c, P, n)$ of $\mathbb{R}^{n}$ with a nonempty interior can be defined by

$$
\mathscr{E}(c, P, n) \triangleq\left\{x \in \mathbb{R}^{n}:(x-c)^{\mathrm{T}} P^{-1}(x-c) \leq 1\right\}
$$

where $c \in \mathbb{R}^{n}$ is the center of $\mathscr{E}(c, P, n)$ and $P>0$ is a positive definite matrix that specifies the ellipsoid's shape and orientation.

Assumption 1: The unknown but bounded noises $w_{k}$ and $v_{k}$ are confined to the following specified ellipsoids:

$$
\begin{gathered}
w_{k} \in \mathscr{E}\left(0, W_{k}, \omega\right) \triangleq\left\{w_{k} \in \mathbb{R}^{\omega}: w_{k}^{\mathrm{T}} W_{k}^{-1} w_{k} \leq 1\right\} \\
v_{k} \in \mathscr{E}\left(0, V_{k}, \nu\right) \triangleq\left\{v_{k} \in \mathbb{R}^{\nu}: v_{k}^{\mathrm{T}} V_{k}^{-1} v_{k} \leq 1\right\}
\end{gathered}
$$

where $W_{k}>0$ and $V_{k}>0$ are known positive definite matrices of appropriate dimensions.

\section{B. Deception attack model}

In this paper, we investigate the following deception attack scenario. Attempting to deteriorate the filtering performance, the adversary injects certain deception signals into the true signals of the control input $u_{k}$ and the measurement outputs $y_{i, k}$ during the process of data transmission through the communication networks. Such an attack scenario can be illustrated by Fig. 1 .

Before giving the deception attack model, we make some further assumptions on the system knowledge that are possessed by the adversary for implementing a successful attack. In this paper, it is assumed that the adversary has sufficient resources and adequate knowledge to arrange a successful attack [25]. Specifically, the adversary, in the first place, knows the accurate values of the control input $u_{k}$ and the measurement output $y_{i, k}$ in real time, and in the second place, has the ability to modify the true values of $u_{k}$ and $y_{i, k}$ to arbitrary ones. Moreover, the attacks are arranged in a coordinated fashion where the deception signals are injected into each communication channel simultaneously to maximize the impact to the plant/estimator [29].

The signals used by the adversary for the deception attacks are generated as follows:

$$
\left\{\begin{array}{l}
\vec{u}_{k}=-u_{k}+\delta_{k} \\
\vec{y}_{i, k}=-y_{i, k}+\vartheta_{i, k}, \quad i=1,2, \ldots, N
\end{array}\right.
$$

where $\delta_{k}$ and $\vartheta_{i, k}(i=1,2, \ldots, N)$ are the unknown but bounded signals belonging to the following ellipsoids:

$$
\begin{gathered}
\delta_{k} \in \mathscr{E}\left(0, S_{k}, m\right) \triangleq\left\{\delta_{k} \in \mathbb{R}^{m}: \delta_{k}^{\mathrm{T}} S_{k}^{-1} \delta_{k} \leq 1\right\}, \\
\vartheta_{i, k} \in \mathscr{E}\left(0, R_{i, k}, p\right) \triangleq\left\{\vartheta_{i, k} \in \mathbb{R}^{p}: \vartheta_{i, k}^{\mathrm{T}} R_{i, k}^{-1} \vartheta_{i, k} \leq 1\right\}
\end{gathered}
$$

with $S_{k}$ and $R_{i, k}(i=1,2, \ldots, N)$ being positive definite matrices of compatible dimensions.

Remark 1: In (7), $\delta_{k}$ and $\vartheta_{i, k}(i=1,2, \ldots, N)$, which have been assumed to be unknown but confined to certain ellipsoidal sets, are used by the adversary to generate the deception attack signals. It should be noted that $\delta_{k}$ and $\vartheta_{i, k}$ have similar forms with the process noise $w_{k}$ and the measurement noise $v_{k}$, and are therefore difficult to be distinguished by the detectors. On the other hand, the most widely implemented attack detector in the practical applications, namely, the $\chi^{2}$ detector, is only effective when the noises obey Gaussian distribution [1]. As such, the utilization of unknown but bounded signals could help to pass through the $\chi^{2}$ detector. In other words, from the adversary's perspective, it is practically reasonable to constrain the malicious signals $\delta_{k}$ and $\vartheta_{i, k}(i=1,2, \ldots, N)$ within given ellipsoidal sets.

Remark 2: In engineering practice, attack detectors are categorized as a software barrier, and there are some other "hard" physical constraints that the adversary would need to face. Such physical constraints include device saturations, bandwidth limitations, channel fading and signal quantizations [6]. The kinds of hardware constraints should be taken into consideration if we are to establish a comprehensive yet realistic deception attack model. On the other hand, such constraints inevitably bring in new challenges that demand new techniques in analyzing the performance and design the filters.

Based on the discussions in Remark 2 and from Fig. 1, we can reformulate the actual control input $\tilde{u}_{k}$ (sent to the plant) and the actual measurement outputs $\tilde{y}_{i, k}$ (fed to the estimator) by

$$
\left\{\begin{array}{l}
\tilde{u}_{k}=u_{k}+\Gamma \vec{u}_{k} \\
\tilde{y}_{i, k}=y_{i, k}+\Xi_{i} \vec{y}_{i, k}, \quad i=1,2, \ldots, N
\end{array}\right.
$$

where the matrices $\Gamma$ and $\Xi_{i}$ represent the physical constraints imposed on the attack signals and are assumed to be of the following forms:

$$
\left\{\begin{array}{l}
\Gamma=\operatorname{diag}\left\{\gamma_{1}, \gamma_{2}, \ldots, \gamma_{m}\right\}, \\
\Xi_{i}=\operatorname{diag}\left\{\xi_{i, 1}, \xi_{i, 2}, \ldots, \xi_{i, p}\right\}, \quad i=1,2, \ldots, N .
\end{array}\right.
$$

Here, the entries of $\Gamma$ and $\Xi_{i}$ have upper- and lower-bounds that are expressed as follows:

$$
\left\{\begin{array}{l}
0 \leq \underline{\gamma}_{j} \leq \gamma_{j} \leq \bar{\gamma}_{j}<\infty, \quad j=1,2, \ldots, m \\
0 \leq \underline{\xi}_{i, s} \leq \xi_{i, s} \leq \bar{\xi}_{i, s}<\infty, \quad s=1,2, \ldots, p
\end{array}\right.
$$

where $0 \leq \underline{\gamma}_{j}<1$ and $\bar{\gamma}_{j} \geq 1$ are known scalars representing the lower- and upper-bounds on $\gamma_{j}$, and $0 \leq \underline{\xi}_{i, s}<1$ and $\bar{\xi}_{i, s} \geq 1$ are known scalars describing the lower- and upperbounds on $\xi_{i, s}$, respectively.

Remark 3: We now take the matrix $\Gamma$ as an example to illustrate how the upper- and lower-bounds on $\gamma_{j}$ affect the behavior of the deception attack signal $\vec{u}_{k}$ (the impact on 
$\vec{y}_{i, k}$ from $\xi_{i, s}$ can be analyzed similarly). Specifically, when $\gamma_{j}=1$, it means that the $j$ th entry of the deception signal $\vec{u}_{k}$ can be injected correctly into the corresponding true $u_{k}$ as the adversary plans, otherwise $\vec{u}_{k}$ might be unexpectedly (from the attacker's perspective) degraded $\left(0 \leq \gamma_{j}<1\right)$ or amplified $\left(\gamma_{j}>1\right)$. In this sense, the model (8)-(10) offers a comprehensive and realistic means to reflect the influence on the attacks resulting from the physical constraints as well as the network-induced complexities.

By denoting

$$
\begin{aligned}
& \underline{\Gamma} \triangleq \operatorname{diag}\left\{\underline{\gamma}_{1}, \underline{\gamma}_{2}, \ldots, \underline{\gamma}_{m}\right\}, \quad \bar{\Gamma} \triangleq \operatorname{diag}\left\{\bar{\gamma}_{1}, \bar{\gamma}_{2}, \ldots, \bar{\gamma}_{m}\right\}, \\
& \Xi_{i} \triangleq \operatorname{diag}\left\{\underline{\xi}_{i, 1}, \underline{\xi}_{i, 2}, \ldots, \underline{\xi}_{i, p}\right\}, \bar{\Xi}_{i} \triangleq \operatorname{diag}\left\{\bar{\xi}_{i, 1}, \bar{\xi}_{i, 2}, \ldots, \bar{\xi}_{i, p}\right\},
\end{aligned}
$$

we can rewrite (10) into the following compact forms:

$$
\left\{\begin{array}{l}
\underline{\Gamma} \leq \Gamma \leq \bar{\Gamma} \\
\Xi_{i} \leq \Xi_{i} \leq \bar{\Xi}_{i}, \quad i=1,2, \ldots, N
\end{array}\right.
$$

In what follows, for the convenience of later derivation, we divide the deception attack signals $\Gamma \vec{u}_{k}$ and $\Xi_{i} \vec{y}_{i, k}$ as follows:

$$
\left\{\begin{array}{l}
\Gamma \vec{u}_{k}=\underline{\Gamma} \vec{u}_{k}+\varphi\left(\vec{u}_{k}\right), \\
\Xi_{i} \vec{y}_{i, k}=\Xi_{i} \vec{y}_{i, k}+\psi_{i}\left(\vec{y}_{i, k}\right), \quad i=1,2, \ldots, N .
\end{array}\right.
$$

It then can be easily checked that

$$
\left\{\begin{array}{l}
\varphi^{\mathrm{T}}\left(\vec{u}_{k}\right)\left(\varphi\left(\vec{u}_{k}\right)-\tilde{\Gamma} \vec{u}_{k}\right) \leq 0, \\
\psi_{i}^{\mathrm{T}}\left(\vec{y}_{i, k}\right)\left(\psi_{i}\left(\vec{y}_{i, k}\right)-\tilde{\Xi}_{i} \vec{y}_{i, k}\right) \leq 0, \quad i=1,2, \ldots, N
\end{array}\right.
$$

where $\tilde{\Gamma} \triangleq \bar{\Gamma}-\underline{\Gamma}>0$ and $\tilde{\Xi}_{i} \triangleq \bar{\Xi}_{i}-\Xi_{i}>0$ are positive definite matrices. Clearly, it follows from Definition 1 that $\varphi\left(\vec{u}_{k}\right)$ and $\psi_{i}\left(\vec{y}_{i, k}\right)$ are vector-valued nonlinear functions satisfying the sector condition and belonging to the sectors $[0, \tilde{\Gamma}]$ and $\left[0, \tilde{\Xi}_{i}\right](i=1,2, \ldots, N)$, respectively.

\section{Design objective}

On account of the deception attacks discussed above, the original system (1)-(2) should be reformulated by

$$
\left\{\begin{aligned}
x_{k+1} & =\left(A_{k}+\sum_{l=1}^{q} \alpha_{l, k} A_{l, k}\right) x_{k}+B_{k} \tilde{u}_{k}+D_{k} w_{k}, \\
\tilde{u}_{k} & =u_{k}+\Gamma \vec{u}_{k}, \\
\tilde{y}_{i, k} & =y_{i, k}+\Xi_{i} \vec{y}_{i, k}, \quad i=1,2, \ldots, N .
\end{aligned}\right.
$$

For the system (14), at each sensing node $i \quad(i=$ $1,2, \ldots, N)$, the following filter structure is adopted:

$$
\hat{x}_{i, k+1}=G_{i, k} \hat{x}_{i, k}+B_{k} u_{k}+\sum_{j \in \mathscr{N}_{i}} \theta_{i j} K_{i j, k}\left(\tilde{y}_{i, k}-C_{i, k} \hat{x}_{i, k}\right)
$$

where $\hat{x}_{i, k} \in \mathbb{R}^{n}$ is the estimate of the state $x_{k}$ based on the $i$ th sensing node, and the matrices $G_{i, k}$ and $K_{i j, k}(i, j=$ $1,2, \ldots, N)$ are the filter parameters to be determined.

Assumption 2: The initial state $x_{0}$ of the system (14) and its estimate values from each sensing node, namely, $\hat{x}_{i, 0}(i=$ $1,2, \ldots, N)$, satisfy:

$$
\mathbb{E}\left\{\left(x_{0}-\hat{x}_{i, 0}\right)\left(x_{0}-\hat{x}_{i, 0}\right)^{\mathrm{T}}\right\} \leq \Phi_{0}
$$

where $\Phi_{0}>0$ is a known positive definite matrix.
The distributed filtering problem under investigation is to estimate the state of the system (1) using a network of filters connected according to the graph $\mathscr{G}$ with the guarantee of variance constraints on the estimation errors. Specifically, the objective of this paper is twofold. For the system (1)-(2) subject to the deception attacks (6), let the communication graph $\mathscr{G}$ and the sequence of positive definite matrices $\left\{\Phi_{k}\right\}_{k \geq 0}$ (prespecified constraints on the estimation error variance) be given. It is our first aim to design the sequences of filtering parameters $\left\{G_{i, k}\right\}_{k \geq 0}$ and $\left\{K_{i j, k}\right\}_{k \geq 0}$ in (15) subject to the given couple $\left(\mathscr{G},\left\{\Phi_{k}\right\}_{k \geq 0}\right)$ such that the following inequalities are satisfied for all $k \geq 0$ :

$$
\mathbb{E}\left\{\left(x_{k}-\hat{x}_{i, k}\right)\left(x_{k}-\hat{x}_{i, k}\right)^{\mathrm{T}}\right\} \leq \Phi_{k}, \quad i=1,2, \ldots, N .
$$

Secondly, within the proposed framework, an optimization problem will be considered for minimizing $\Phi_{k}$ in the sense of matrix trace at each time instant to ensure the locally optimal filtering performance. This problem will be referred to as a variance-constrained distributed filtering problem subject to deception attacks.

\section{Distributed Filter Design}

In this section, we will design a distributed filter of form (15) for system (1)-(2) subject to multiplicative noises, unknown but bounded disturbances and deception attacks. A sufficient condition for the existence of the desired filter will be formulated in terms of a set of recursive linear matrix inequalities (RLMIs). First, two lemmas which are useful for our subsequent development are introduced as follows.

Lemma 1: (Schur Complement Lemma) Given constant matrices $\mathcal{S}_{1}, \mathcal{S}_{2}, \mathcal{S}_{3}$ where $\mathcal{S}_{1}=\mathcal{S}_{1}^{\mathrm{T}}$ and $0<\mathcal{S}_{2}=\mathcal{S}_{2}^{\mathrm{T}}$, then $\mathcal{S}_{1}+\mathcal{S}_{3}^{\mathrm{T}} \mathcal{S}_{2}^{-1} \mathcal{S}_{3}<0$ if and only if

$$
\left[\begin{array}{cc}
\mathcal{S}_{1} & \mathcal{S}_{3}^{\mathrm{T}} \\
\mathcal{S}_{3} & -\mathcal{S}_{2}
\end{array}\right]<0, \quad \text { or } \quad\left[\begin{array}{cc}
-\mathcal{S}_{2} & \mathcal{S}_{3} \\
\mathcal{S}_{3}^{\mathrm{T}} & \mathcal{S}_{1}
\end{array}\right]<0 .
$$

Lemma 2: (S-procedure [2]) Let $\kappa_{0}(\cdot), \kappa_{1}(\cdot), \ldots, \kappa_{s}(\cdot)$ be quadratic functions of the variable $\zeta \in \mathbb{R}^{n}: \kappa_{j}(\zeta) \triangleq \zeta^{\mathrm{T}} T_{j} \zeta$ $(j=0,1, \ldots, s)$, where $T_{j}^{\mathrm{T}}=T_{j}$. If there exist $\tau_{1} \geq 0, \ldots$, $\tau_{s} \geq 0$ such that $T_{0}-\sum_{j=1}^{s} \tau_{j} T_{j} \leq 0$, then the following is true:

$$
\kappa_{1}(\zeta) \leq 0, \ldots, \kappa_{s}(\zeta) \leq 0 \Longrightarrow \kappa_{0}(\zeta) \leq 0
$$

From the system (14) and the filter (15), for sensing node $i(i=1,2, \ldots, N)$, the one-step ahead estimation error is calculated by

$$
\begin{aligned}
& x_{k+1}-\hat{x}_{i, k+1} \\
= & \left(A_{k}+\sum_{l=1}^{q} \alpha_{l, k} A_{l, k}\right) x_{k}-B_{k} \underline{\Gamma} u_{k}+B_{k} \underline{\Gamma} \delta_{k}+B_{k} \varphi\left(\vec{u}_{k}\right) \\
& +D_{k} w_{k}-G_{i, k} \hat{x}_{i, k}-\sum_{j \in \mathscr{N}_{i}} \theta_{i j} K_{i j, k}\left(I-\underline{\Xi}_{i}\right) C_{i, k} x_{k} \\
& -\sum_{j \in \mathscr{N}_{i}} \theta_{i j} K_{i j, k}\left(I-\Xi_{i}\right) E_{i, k} v_{k}-\sum_{j \in \mathscr{N}_{i}} \theta_{i j} K_{i j, k} \Xi_{i} \vartheta_{i, k} \\
& -\sum_{j \in \mathscr{N}_{i}} \theta_{i j} K_{i j, k} \psi_{i}\left(\vec{y}_{i, k}\right)+\sum_{j \in \mathscr{N}_{i}} \theta_{i j} K_{i j, k} C_{i, k} \hat{x}_{i, k} .
\end{aligned}
$$


For simplicity of the further development, we denote

$$
\begin{aligned}
& \mathcal{T}_{\lambda, i} \triangleq[\underbrace{0 \cdots 0}_{i-1} I_{\lambda} \underbrace{0 \cdots 0}_{N-i}], \\
& \Omega_{\lambda, i} \triangleq \mathcal{T}_{\lambda, i}^{\mathrm{T}} \mathcal{T}_{\lambda, i}\left(I_{N} \otimes I_{\lambda}\right), \quad \lambda=\{n, q, p\}, i=1, \ldots, N .
\end{aligned}
$$

Denoting $\tilde{x}_{i, k} \triangleq x_{k}-\hat{x}_{i, k}$, we can rewrite (20) into a compact form as follows:

$$
\begin{aligned}
\tilde{x}_{k+1}= & \left(\mathcal{A}_{k}+\bar{\alpha}_{k} \overline{\mathcal{A}}_{k}\right) \zeta_{k}-\mathcal{B}_{k} \mu_{k}+\mathcal{B}_{k} \tilde{\delta}_{k}+\overline{\mathcal{B}}_{k} \tilde{\varphi}_{k} \\
& +\mathcal{D}_{k} \tilde{w}_{k}-\mathcal{G}_{k} \hat{x}_{k}-\mathcal{K}_{k}(I-\Xi) \mathcal{C}_{k} \zeta_{k} \\
& -\mathcal{K}_{k}(I-\Xi) \mathcal{E}_{k} \tilde{v}_{k}-\mathcal{K}_{k} \Xi \vartheta_{k} \\
& -\mathcal{K}_{k} \psi_{k}+\mathcal{K}_{k} \mathcal{C}_{k} \hat{x}_{k}
\end{aligned}
$$

where

$\tilde{x}_{k} \triangleq \operatorname{col}_{N}\left\{\tilde{x}_{i, k}\right\}, \zeta_{k} \triangleq \operatorname{col}_{N}\left\{x_{k}\right\}, \hat{x}_{k} \triangleq \operatorname{col}_{N}\left\{\hat{x}_{i, k}\right\}$, $\mu_{k} \triangleq \operatorname{col}_{N}\left\{u_{k}\right\}, \tilde{\delta}_{k} \triangleq \operatorname{col}_{N}\left\{\delta_{k}\right\}, \tilde{\varphi}_{k} \triangleq \operatorname{col}_{N}\left\{\varphi\left(\vec{u}_{k}\right)\right\}$,

$\tilde{w}_{k} \triangleq \operatorname{col}_{N}\left\{w_{k}\right\}, \tilde{v}_{k} \triangleq \operatorname{col}_{N}\left\{v_{k}\right\}, \vartheta_{k} \triangleq \operatorname{col}_{N}\left\{\vartheta_{i, k}\right\}$,

$\psi_{k} \triangleq \operatorname{col}_{N}\left\{\psi_{i}\left(\vec{y}_{i, k}\right)\right\}, \mathcal{A}_{k} \triangleq \operatorname{diag}_{N}\left\{A_{k}\right\}$,

$\tilde{\mathcal{A}}_{k} \triangleq \operatorname{diag}_{N}\left\{\sum_{l=1}^{q} A_{l, k}\right\}, \alpha_{k} \triangleq\left[\begin{array}{lll}\alpha_{1, k} I & \cdots & \alpha_{q, k} I\end{array}\right]$,

$\bar{A}_{k} \triangleq\left[\begin{array}{llll}A_{1, k}^{\mathrm{T}} & A_{2, k}^{\mathrm{T}} & \cdots & A_{q, k}^{\mathrm{T}}\end{array}\right]^{\mathrm{T}}, \bar{\alpha}_{k} \triangleq \operatorname{diag}_{N}\left\{\alpha_{k}\right\}$,

$\overline{\mathcal{A}}_{k} \triangleq \operatorname{diag}_{N}\left\{\bar{A}_{k}\right\}, \mathcal{B}_{k} \triangleq \operatorname{diag}_{N}\left\{B_{k} \underline{\Gamma}\right\}, \overline{\mathcal{B}}_{k} \triangleq \operatorname{diag}_{N}\left\{B_{k}\right\}$,

$\mathcal{C}_{k} \triangleq \operatorname{diag}_{N}\left\{C_{i, k}\right\}, \mathcal{D}_{k} \triangleq \operatorname{diag}_{N}\left\{D_{k}\right\}, \mathcal{E}_{k} \triangleq \operatorname{diag}_{N}\left\{E_{i, k}\right\}$,

$\mathcal{G}_{k} \triangleq \operatorname{diag}_{N}\left\{G_{i, k}\right\}, \Xi \triangleq \operatorname{diag}_{N}\left\{\Xi_{i}\right\}, \mathcal{K}_{k} \triangleq\left[\theta_{i j} K_{i j, k}\right]_{N \times N}$.

Noticing that when $j \notin \mathscr{N}_{i}, \theta_{i j}=0$, we know that $\mathcal{K}_{k}$ is a sparse matrix which can be described by

$$
\mathcal{K}_{k} \in \mathscr{Q}_{n \times m}
$$

where $\mathscr{Q}_{n \times m} \triangleq\left\{\mathcal{Q}=\left[Q_{i j}\right] \in \mathbb{R}^{n N \times m N} \mid Q_{i j} \in\right.$ $\mathbb{R}^{n \times m,}, Q_{i j}=0$ if $\left.j \notin \mathscr{N}_{i}\right\}$.

The following theorem presents a sufficient condition for the existence of the desired distributed filter by RLMI approach.

Theorem 1: For the system (1)-(2) subject to the deception attacks (6), let the network topology $\mathscr{G}$ and the prespecified sequence of variance constraints $\left\{\Phi_{k}\right\}_{k \geq 0}$ be given. The design objective (17) is achieved if there exist sequences of real-valued matrices $\left\{\mathcal{G}_{k}\right\}_{k>0}$ and $\left\{\mathcal{K}_{k}\right\}_{k>0}\left(\mathcal{K}_{k} \in\right.$ $\left.\mathscr{Q}_{n \times m}\right)$, sequences of positive definite matrices $\left\{\tilde{S}_{k}\right\}_{k \geq 0}$ and $\left\{\tilde{R}_{i, k}\right\}_{k \geq 0}$, sequences of non-negative scalars $\left\{\tau_{1, k}\right\}_{k \geq 0}$, $\left\{\tau_{2, k}\right\}_{k \geq 0},\left\{\tau_{3, k}\right\}_{k \geq 0},\left\{\tau_{4, k}\right\}_{k \geq 0},\left\{\epsilon_{i, k}\right\}_{k \geq 0},\left\{\varrho_{i, k}\right\}_{k \geq 0}$ and $\left\{\rho_{i, k}\right\}_{k>0} \quad(i=1,2, \ldots, N)$ satisfying the following $N$ RLMIs:

$$
\left[\begin{array}{ccc}
-\Delta_{k} & * & * \\
\mathcal{T}_{n, i} \bar{\Lambda}_{k} & -\Phi_{k+1} & * \\
\mathcal{T}_{n, i} \hat{\Lambda}_{k} & 0 & -\Phi_{k+1}
\end{array}\right] \leq 0, \quad i=1,2, \ldots, N
$$

where

$$
\begin{aligned}
\Delta_{k} & \triangleq \tau_{4, k} \Pi_{k}+\sum_{i=1}^{N} \rho_{i, k} \Upsilon_{i, k} \\
& +\operatorname{diag}\left\{1-\sum_{i=1}^{N}\left(\epsilon_{i, k}+\varrho_{i, k}\right)-\tau_{1, k}-\tau_{2, k}-\tau_{3, k},\right.
\end{aligned}
$$

$$
\begin{aligned}
& \sum_{i=1}^{N} \epsilon_{i, k} \mathcal{T}_{q, i}^{\mathrm{T}} \mathcal{T}_{q, i}, \tilde{S}_{k}, \sum_{i=1}^{N} \mathcal{T}_{p, i}^{\mathrm{T}} \tilde{R}_{i, k} \mathcal{T}_{p, i}, \\
& \left.\tau_{2, k} W_{k}^{-1}, \tau_{3, k} V_{k}^{-1}, 0,0\right\}, \\
& \bar{\Pi}_{k} \triangleq\left[\begin{array}{c}
u_{k}^{\mathrm{T}} \tilde{\Gamma} \\
0 \\
-\tilde{\Gamma} \\
0 \\
0 \\
0 \\
2 I_{m} \\
0
\end{array}\right], \bar{\Upsilon}_{i, k} \triangleq\left[\begin{array}{c}
\hat{x}_{k}^{\mathrm{T}} \Omega_{n, i} \operatorname{diag}_{N}\left\{C_{i, k}^{\mathrm{T}} \tilde{\Xi}_{i}\right\} \Omega_{p, i} \\
\Omega_{q, i} \operatorname{diag}_{N}\left\{P_{k}^{\mathrm{T}} C_{i, k}^{\mathrm{T}} \tilde{\Xi}_{i}\right\} \Omega_{p, i} \\
0 \\
-\Omega_{p, i} \operatorname{diag}_{N}\left\{\tilde{\Xi}_{i}\right\} \Omega_{p, i} \\
0 \\
\mathcal{T}_{p, i} \operatorname{diag}_{N}\left\{E_{i, k}^{\mathrm{T}} \tilde{\Xi}_{i}\right\} \Omega_{p, i} \\
0 \\
2 \Omega_{p, i} I_{p N} \Omega_{p, i}
\end{array}\right] \\
& \Pi_{k} \triangleq \frac{1}{2}\left[\begin{array}{llllllll}
0 & 0 & 0 & 0 & 0 & 0 & \bar{\Pi}_{k} & 0
\end{array}\right], \\
& \Upsilon_{i, k} \triangleq \frac{1}{2}\left[\begin{array}{llllllll}
0 & 0 & 0 & 0 & 0 & 0 & 0 & \bar{\Upsilon}_{i, k}
\end{array}\right] \text {, } \\
& \mathscr{I}_{m} \triangleq \mathbf{1}_{N} \otimes I_{m}, \quad \mathscr{I}_{\omega} \triangleq \mathbf{1}_{N} \otimes I_{\omega}, \quad \mathscr{I}_{\nu} \triangleq \mathbf{1}_{N} \otimes I_{\nu}, \\
& \bar{\Lambda}_{11} \triangleq\left(\mathcal{A}_{k}-\mathcal{G}_{k}+\mathcal{K}_{k} \Xi \mathcal{C}_{k}\right) \hat{x}_{k}-\mathcal{B}_{k} \mu_{k}, \\
& \bar{\Lambda}_{12} \triangleq \mathcal{A}_{k} \mathcal{P}_{k}-\mathcal{K}_{k}(I-\Xi) \mathcal{C}_{k} \mathcal{P}_{k}, \\
& \bar{\Lambda}_{k} \triangleq\left[\begin{array}{lllll}
\bar{\Lambda}_{11} & \bar{\Lambda}_{12} & \mathcal{B}_{k} \mathscr{I}_{m} & -\mathcal{K}_{k} \Xi & \mathcal{D}_{k} \mathscr{I}_{\omega}
\end{array}\right. \\
& \left.-\mathcal{K}_{k}(I-\Xi) \mathcal{E}_{k} \mathscr{I}_{\nu} \quad \overline{\mathcal{B}}_{k} \mathscr{I}_{m} \quad-\mathcal{K}_{k}\right], \\
& \hat{\Lambda}_{k} \triangleq\left[\begin{array}{llllllll}
\tilde{\mathcal{A}}_{k} \hat{x}_{k} & \tilde{\mathcal{A}}_{k} \mathcal{P}_{k} & 0 & 0 & 0 & 0 & 0 & 0
\end{array}\right]
\end{aligned}
$$

with $P_{k}$ being a factorization of $\Phi_{k}$ (i.e., $\Phi_{k}=P_{k} P_{k}^{\mathrm{T}}$ ) and $\mathcal{P}_{k} \triangleq \operatorname{diag}_{N}\left\{P_{k}\right\}$. Moreover, the parameters $S_{k}$ and $R_{i, k}$ can be computed by $S_{k}=\tau_{1, k} \tilde{S}_{k}^{-1}$ and $R_{i, k}=\varrho_{i, k} \tilde{R}_{i, k}^{-1}$.

Proof: We prove Theorem 1 by induction which can be divided into two steps, namely, the initial step and the inductive step.

Initial step. For $k=0$, it can be known directly from Assumption 2 that the initial value of the state $x_{0}$ and its estimates $\hat{x}_{i, 0}(i=1,2, \ldots, N)$ satisfy

$$
\mathbb{E}\left\{\left(x_{0}-\hat{x}_{i, 0}\right)\left(x_{0}-\hat{x}_{i, 0}\right)^{\mathrm{T}}\right\} \leq \Phi_{0} .
$$

Inductive step. Given that at the time instant $k>0$, the following is true:

$$
\mathbb{E}\left\{\left(x_{k}-\hat{x}_{i, k}\right)\left(x_{k}-\hat{x}_{i, k}\right)^{\mathrm{T}}\right\} \leq \Phi_{k},
$$

then we aim to, with the condition given in the theorem, demonstrate that the following set of inequalities holds for all $i$ :

$$
\mathbb{E}\left\{\left(x_{k+1}-\hat{x}_{i, k+1}\right)\left(x_{k+1}-\hat{x}_{i, k+1}\right)^{\mathrm{T}}\right\} \leq \Phi_{k+1} .
$$

Since the inequality (31) is true, it follows from [9] that there exists a sequence of vectors $z_{i, k} \in \mathbb{R}^{q}$ (with $\left.\mathbb{E}\left\{z_{i, k}^{\mathrm{T}} z_{i, k}\right\} \leq 1\right)$ satisfying $x_{k}=\hat{x}_{i, k}+P_{k} z_{i, k}$ where $P_{k} \in \mathbb{R}^{n \times q}$ is a factorization of $\Phi_{k}=P_{k} P_{k}^{\mathrm{T}}$. Denoting $z_{k} \triangleq \operatorname{col}_{N}\left\{z_{i, k}\right\}$ and noticing $\mathcal{P}_{k}=\operatorname{diag}_{N}\left\{P_{k}\right\}$, we can further acquire that

$$
\zeta_{k}=\hat{x}_{k}+\mathcal{P}_{k} z_{k}
$$

Now, substituting (33) into (21) yields

$\tilde{x}_{k+1}=\left(\mathcal{A}_{k}+\bar{\alpha}_{k} \overline{\mathcal{A}}_{k}-\mathcal{G}_{k}-\mathcal{K}_{k}(I-\Xi) \mathcal{C}_{k}+\mathcal{K}_{k} \mathcal{C}_{k}\right) \hat{x}_{k}$ 


$$
\begin{aligned}
& -\mathcal{B}_{k} \mu_{k}+\left(\left(\mathcal{A}_{k}+\bar{\alpha}_{k} \overline{\mathcal{A}}_{k}\right) \mathcal{P}_{k}-\mathcal{K}_{k}(I-\Xi) \mathcal{C}_{k} \mathcal{P}_{k}\right) z_{k} \\
& +\mathcal{B}_{k} \mathscr{I}_{m} \delta_{k}+\overline{\mathcal{B}}_{k} \mathscr{I}_{m} \varphi_{k}+\mathcal{D}_{k} \mathscr{I}_{\omega} w_{k} \\
& -\mathcal{K}_{k}(I-\Xi) \mathcal{E}_{k} \mathscr{I}_{\nu} v_{k}-\mathcal{K}_{k} \Xi \vartheta_{k}-\mathcal{K}_{k} \psi_{k} .
\end{aligned}
$$

We now define a vector as follows:

$$
\beta_{k} \triangleq\left[\begin{array}{llllllll}
1 & z_{k}^{\mathrm{T}} & \delta_{k}^{\mathrm{T}} & \vartheta_{k}^{\mathrm{T}} & w_{k}^{\mathrm{T}} & v_{k}^{\mathrm{T}} & \varphi_{k}^{\mathrm{T}} & \psi_{k}^{\mathrm{T}}
\end{array}\right]^{\mathrm{T}} .
$$

Then, the one-step ahead estimation error $\tilde{x}_{k+1}$ in (34) is expressed by

$$
\tilde{x}_{k+1}=\Lambda_{k} \beta_{k}
$$

where

$$
\begin{aligned}
& \Lambda_{11} \triangleq\left(\mathcal{A}_{k}+\bar{\alpha}_{k} \overline{\mathcal{A}}_{k}-\mathcal{G}_{k}+\mathcal{K}_{k} \Xi \mathcal{C}_{k}+\mathcal{G}_{k}\right) \hat{x}_{k}-\mathcal{B}_{k} \mu_{k}, \\
& \Lambda_{12} \triangleq\left(\mathcal{A}_{k}+\bar{\alpha}_{k} \overline{\mathcal{A}}_{k}\right) \mathcal{P}_{k}-\mathcal{K}_{k}(I-\Xi) \mathcal{C}_{k} \mathcal{P}_{k}+\mathcal{G}_{k} \mathcal{P}_{k}, \\
& \Lambda_{k} \triangleq\left[\begin{array}{lllll}
\Lambda_{11} & \Lambda_{12} & \mathcal{B}_{k} \mathscr{I}_{m} & -\mathcal{K}_{k} \Xi & \mathcal{D}_{k} \mathscr{I}_{\omega}
\end{array}\right. \\
& \left.-\mathcal{K}_{k}(I-\Xi) \mathcal{E}_{k} \mathscr{I}_{\nu} \quad \overline{\mathcal{B}}_{k} \mathscr{I}_{m} \quad-\mathcal{K}_{k}\right] .
\end{aligned}
$$

Next, we decompose the matrix $\Lambda_{k}$ into a deterministic part $\bar{\Lambda}_{k}$ defined in (28) and a stochastic part $\tilde{\Lambda}_{k}$ which contains the random variable $\bar{\alpha}_{k}$ as follows:

$$
\Lambda_{k}=\bar{\Lambda}_{k}+\tilde{\Lambda}_{k}
$$

where

$$
\tilde{\Lambda}_{k} \triangleq\left[\begin{array}{lllllllll}
\bar{\alpha}_{k} \overline{\mathcal{A}}_{k} \hat{x}_{k} & \bar{\alpha}_{k} \overline{\mathcal{A}}_{k} \mathcal{P}_{k} & 0 & 0 & 0 & 0 & 0 & 0
\end{array}\right] .
$$

Then, by taking into consideration the statistical properties of the random variable $\bar{\alpha}_{k}$, we have

$$
\begin{aligned}
& \mathbb{E}\left\{\Lambda_{k}^{\mathrm{T}} \mathcal{T}_{n, i}^{\mathrm{T}} \Phi_{k+1}^{-1} \mathcal{T}_{n, i} \Lambda_{k}\right\} \\
= & \bar{\Lambda}_{k}^{\mathrm{T}} \mathcal{T}_{n, i}^{\mathrm{T}} \Phi_{k+1}^{-1} \mathcal{T}_{n, i} \bar{\Lambda}_{k}+\hat{\Lambda}_{k}^{\mathrm{T}} \mathcal{T}_{n, i}^{\mathrm{T}} \Phi_{k+1}^{-1} \mathcal{T}_{n, i} \hat{\Lambda}_{k} .
\end{aligned}
$$

In the following, we shall proceed to deal with the constraints (13) imposed on the attack signals $\vec{u}_{k}$ and $\vec{y}_{i, k}(i=$ $1,2, \ldots, N)$. It can be known from (13) that the nonlinear vector-valued functions $\varphi\left(\vec{u}_{k}\right)$ and $\psi_{i}\left(\vec{y}_{i_{2} k}\right)(i=1,2, \ldots, N)$ are belonging to the sectors $[0, \tilde{\Gamma}]$ and $\left[0, \Xi_{i}\right](i=1,2, \ldots, N)$, respectively. As such, we can perform the following derivations:

$$
\varphi^{\mathrm{T}}\left(\vec{u}_{k}\right)\left(\varphi\left(\vec{u}_{k}\right)-\tilde{\Gamma} \vec{u}_{k}\right) \leq 0 \Longleftrightarrow \beta_{k}^{\mathrm{T}} \Pi_{k} \beta_{k} \leq 0
$$

where $\Pi_{k}$ is defined in (25).

Likewise, we have

$$
\beta_{k}^{\mathrm{T}} \Upsilon_{i, k} \beta_{k} \leq 0
$$

where $\Upsilon_{i, k}$ is defined in (26).

For the brevity of presentation, we denote

$$
\begin{aligned}
N_{i, k} & \triangleq \operatorname{diag}\left\{-1, \mathcal{T}_{q, i}^{\mathrm{T}} \mathcal{T}_{q, i}, 0,0,0,0,0,0\right\}, \\
M_{1, k} & \triangleq \operatorname{diag}\left\{-1,0, S_{k}^{-1}, 0,0,0,0,0\right\}, \\
J_{i, k} & \triangleq \operatorname{diag}\left\{-1,0,0, \mathcal{T}_{p, i}^{\mathrm{T}} R_{i, k}^{-1} \mathcal{T}_{p, i}, 0,0,0,0\right\}, \\
M_{2, k} & \triangleq \operatorname{diag}\left\{-1,0,0,0, W_{k}^{-1}, 0,0,0\right\}, \\
M_{3, k} & \triangleq \operatorname{diag}\left\{-1,0,0,0,0, V_{k}^{-1}, 0,0\right\} .
\end{aligned}
$$

According to Definition 2, it is not difficult to reformulate the constraints $\mathbb{E}\left\{z_{i, k}^{\mathrm{T}} z_{i, k}\right\} \leq 1, \delta_{k} \in \mathscr{E}\left(0, S_{k}, m\right), \vartheta_{i, k} \in$
$\mathscr{E}\left(0, R_{i, k}, p\right), w_{k} \in \mathscr{E}\left(0, W_{k}, \omega\right)$ and $v_{k} \in \mathscr{E}\left(0, V_{k}, \nu\right)$ in terms of the variable $\beta_{k}$ as follows:

$$
\begin{aligned}
& \beta_{k}^{\mathrm{T}} N_{i, k} \beta_{k} \leq 0, \beta_{k}^{\mathrm{T}} M_{1, k} \beta_{k} \leq 0, \beta_{k}^{\mathrm{T}} J_{i, k} \beta_{k} \leq 0, \\
& \beta_{k}^{\mathrm{T}} M_{2, k} \beta_{k} \leq 0, \beta_{k}^{\mathrm{T}} M_{3, k} \beta_{k} \leq 0 .
\end{aligned}
$$

By applying the Schur Complement Lemma (Lemma 1) to the set of RLMIs (23) and noting that $S_{k}=\tau_{1, k} \tilde{S}_{k}^{-1}$ and $R_{i, k}=\varrho_{i, k} \tilde{R}_{i, k}^{-1}$, we obtain

$$
\bar{\Lambda}_{k}^{\mathrm{T}} \mathcal{T}_{n, i}^{\mathrm{T}} \Phi_{k+1}^{-1} \mathcal{T}_{n, i} \bar{\Lambda}_{k}+\hat{\Lambda}_{k}^{\mathrm{T}} \mathcal{T}_{n, i}^{\mathrm{T}} \Phi_{k+1}^{-1} \mathcal{T}_{n, i} \hat{\Lambda}_{k}-\Delta_{k} \leq 0
$$

which, by (24), is equivalent to

$$
\begin{aligned}
& \bar{\Lambda}_{k}^{\mathrm{T}} \mathcal{T}_{n, i}^{\mathrm{T}} \Phi_{k+1}^{-1} \mathcal{T}_{n, i} \bar{\Lambda}_{k}+\hat{\Lambda}_{k}^{\mathrm{T}} \mathcal{T}_{n, i}^{\mathrm{T}} \Phi_{k+1}^{-1} \mathcal{T}_{n, i} \hat{\Lambda}_{k} \\
- & \operatorname{diag}\{1,0,0,0,0,0,0,0\} \\
- & \tau_{1, k} M_{1, k}-\tau_{2, k} M_{2, k}-\tau_{3, k} M_{3, k}-\tau_{4, k} \Pi_{k} \\
& -\sum_{i=1}^{N} \epsilon_{i, k} N_{i, k}-\sum_{i=1}^{N} \rho_{i, k} \Upsilon_{i, k}-\sum_{i=1}^{N} \varrho_{i, k} J_{i, k} \leq 0 .
\end{aligned}
$$

By resorting to Lemma 2 and on account of (40), (41) and (42), we immediately arrive at

$$
\beta_{k}^{\mathrm{T}}\left(\bar{\Lambda}_{k}^{\mathrm{T}} \mathcal{T}_{n, i}^{\mathrm{T}} \Phi_{k+1}^{-1} \mathcal{T}_{n, i} \bar{\Lambda}_{k}+\hat{\Lambda}_{k}^{\mathrm{T}} \mathcal{T}_{n, i}^{\mathrm{T}} \Phi_{k+1}^{-1} \mathcal{T}_{n, i} \hat{\Lambda}_{k}\right) \beta_{k} \leq 1,
$$

which, by means of (39), further indicates that

$$
\mathbb{E}\left\{\beta_{k}^{\mathrm{T}} \Lambda_{k}^{\mathrm{T}} \mathcal{T}_{n, i}^{\mathrm{T}} \Phi_{k+1}^{-1} \mathcal{T}_{n, i} \Lambda_{k} \beta_{k}\right\} \leq 1,
$$

or equivalently,

$$
\mathbb{E}\left\{\tilde{x}_{i, k+1}^{\mathrm{T}} \Phi_{k+1}^{-1} \tilde{x}_{i, k+1}\right\} \leq 1 .
$$

By using Schur Complement Lemma again, the inequality (47) holds if and only if

$$
\mathbb{E}\left\{\tilde{x}_{i, k+1} \tilde{x}_{i, k+1}^{\mathrm{T}}\right\} \leq \Phi_{k+1},
$$

which accomplishes the induction. Accordingly, we conclude that the design objective (17) is achieved subject to the fixed communication topology $\mathscr{G}$ and variance constraints $\left\{\Phi_{k}\right\}_{k \geq 0}$. The desired sequences of filtering parameters $\left\{\mathcal{G}_{k}\right\}_{k \geq 0}$ and $\left\{\mathcal{K}_{k}\right\}_{k \geq 0}$ can be obtained by solving the set of RLMIs (23) iteratively. The proof is now complete.

In the following stage, an optimization problem is formulated with the purpose to determine the filtering gains ensuring the locally optimal filtering performance by minimizing $\Phi_{k}$ in the sense of matrix trace at each time instant.

Corollary 1: For the system (1)-(2) subject to the deception attacks (6), let the network topology $\mathscr{G}$ be given. A sequence of minimized $\left\{\Phi_{k}\right\}_{k \geq 1}$ can be guaranteed (in the sense of matrix trace) if there exist sequences of real-valued matrices $\left\{\mathcal{G}_{k}\right\}_{k \geq 0}$ and $\left\{\mathcal{K}_{k}\right\}_{k \geq 0}\left(\mathcal{K}_{k} \in \mathscr{Q}_{n \times m}\right)$, sequences of positive definite matrices $\left\{\tilde{S}_{k}\right\}_{k \geq 0}$ and $\left\{\tilde{R}_{i, k}\right\}_{k \geq 0}$, sequences of nonnegative scalars $\left\{\tau_{1, k}\right\}_{k \geq 0},\left\{\tau_{2, k}\right\}_{k \geq 0},\left\{\tau_{3, k}\right\}_{k \geq 0},\left\{\tau_{4, k}\right\}_{k \geq 0}$, $\left\{\epsilon_{i, k}\right\}_{k \geq 0},\left\{\varrho_{i, k}\right\}_{k \geq 0}$ and $\left\{\rho_{i, k}\right\}_{k \geq 0}(i=1,2, \ldots, N)$ solving the following optimization problem:

$$
\begin{gathered}
\min _{\Phi_{k+1}, \mathcal{G}_{k}, \mathcal{K}_{k}, \tilde{S}_{k}, \tilde{R}_{i, k}, \tau_{1, k}, \tau_{2, k}, \tau_{3, k}, \tau_{4, k}, \epsilon_{i, k}, \varrho_{i, k}, \rho_{i, k}} \operatorname{tr}\left[\Phi_{k+1}\right] \\
\text { subject to }\left[\begin{array}{ccc}
-\Delta_{k} & * & * \\
\mathcal{T}_{n, i} \bar{\Lambda}_{k} & -\Phi_{k+1} & * \\
\mathcal{T}_{n, i} \hat{\Lambda}_{k} & 0 & -\Phi_{k+1}
\end{array}\right] \leq 0 .
\end{gathered}
$$


Remark 4: So far, the addressed variance-constrained distributed filtering problem has been discussed for the timevarying systems with multiplicative noises, unknown but bounded disturbances and deception attacks. The existence condition of the desired distributed filter has been established by resorting to the recursive linear matrix inequality approach. The filter gains can be determined via solving a set of RLMIs iteratively. The optimization problem for the locally optimal filtering performance has also been solved by minimizing the constraints imposed on the estimation error variance in the sense of matrix trace. One of our future research topics is the variance-constrained distributed filtering subject to other frequently seen network-induced complexities such as transmission delay [4], [20], [37], multiple missing measurements [15], [22] and quantization effects [19], [35], [36]. It is also our interest to apply the proposed technique to deal with the consensus control problems for stochastic multi-agent systems studied in [17], where the open communication network may be also under attacks.

\section{NUMERICAL EXAMPLE}

In this section, an illustrative example is presented to illustrate the effectiveness of the proposed algorithm. We consider a target tracking system whose dynamic model is given as follows:

$$
x_{k+1}=\left[\begin{array}{ll}
1 & T \\
0 & 1
\end{array}\right]+D w_{k}
$$

where $T$ is the sampling period and the state $x_{k}=\left[\begin{array}{ll}s_{k} & \dot{s}_{k}\end{array}\right]^{\mathrm{T}}$ with $s_{k}$ and $\dot{s}_{k}$ being the position and velocity, respectively. It is worth mentioning in (50), the system parameters are timeinvariant and the state is only subject to the external additive noise $w_{k}$. However, in the real-world application, because of the changeable circumstance, these parameters are usually time-varying. Moreover, the system may contain, apart from the additive noises, certain multiplicative disturbances that have significant impact on the performance [11]. Taking these into account, we propose the model of system (1)-(2) with following parameters that are of more practical significance:

$$
\begin{aligned}
A_{k} & =\left[\begin{array}{ll}
1 & T \\
0 & 1
\end{array}\right], T=0.1, \\
A_{1, k} & =\left[\begin{array}{cc}
0.08+0.1 \sin (3 k) & -0.05 \\
0.01 & -0.01+0.02 \cos (k)
\end{array}\right], \\
A_{2, k} & =\left[\begin{array}{cc}
0.06+0.01 \sin (10 k) & 0.02 \\
-0.01 & -0.07+0.02 \cos (k)
\end{array}\right], \\
C_{1, k} & =\left[\begin{array}{cc}
1 & 0
\end{array}\right], \\
C_{2, k} & =\left[\begin{array}{cc}
0.9+\sin (k) & 0
\end{array}\right], \\
C_{3, k} & =\left[\begin{array}{ll}
0.75+0.5 \sin (k) & 0.1
\end{array}\right], \\
D_{k} & =\left[\begin{array}{c}
0.1+0.05 \cos (3 k) \\
0.2+0.04 \exp \{-k\}
\end{array}\right], \\
E_{1, k} & =0.2+0.05 \cos (3 k), \\
E_{2, k} & =0.2+0.15 \sin (2 k), \\
E_{3, k} & =0.15+0.05 \sin (2 k) .
\end{aligned}
$$

Suppose the unknown but bounded disturbances are $w_{k}=$ $0.2 \sin (5 k)$ and $v_{k}=0.5 \cos (2 k)$ and set $W_{k}=0.04, V_{k}=$
0.25 . Then it can be easily checked that $w_{k}$ and $v_{k}$ belong to the ellipsoid sets defined in (5).

Suppose that there are three sensor nodes connected according to graph $\mathscr{G}$. The associated adjacency matrix $\mathscr{L}$ is selected as follows:

$$
\mathscr{L}=\left[\begin{array}{lll}
1 & 0 & 1 \\
0 & 1 & 1 \\
1 & 1 & 1
\end{array}\right]
$$

Set the initial values as follows:

$$
\begin{aligned}
x_{0} & =\left[\begin{array}{l}
5 \\
3
\end{array}\right], \quad \hat{x}_{1,0}=\left[\begin{array}{l}
2.8 \\
1.6
\end{array}\right], \quad \hat{x}_{2,0}=\left[\begin{array}{l}
3.0 \\
2.0
\end{array}\right], \\
\hat{x}_{3,0} & =\left[\begin{array}{l}
3.2 \\
1.2
\end{array}\right], \quad \Phi_{0}=\left[\begin{array}{cc}
15 & 0.1 \\
0.1 & 15
\end{array}\right] .
\end{aligned}
$$

Then it can be easily checked that (16) is satisfied.

Suppose that the that the constraints imposed on the attack signals are characterized by $\underline{\Gamma}=0.8, \bar{\Gamma}=1.2, \Xi_{1}=0.8$, $\bar{\Xi}_{1}=1.2, \Xi_{2}=0.9, \bar{\Xi}_{2}=1.1, \Xi_{3}=0.7$ and $\bar{\Xi}_{3}=1.3$.

In this section, we proceed to utilize the algorithm proposed in Corollary 1 to solve the optimization problem (49). By using Matlab software, the simulation is carried out and the results are shown in Figs. 2-7. Specifically, Figs. 2-3 depict the trajectories of the individual entries of the system state $x_{k}$ (i.e., $x_{k}^{(1)}$ and $x_{k}^{(2)}$ ) and their estimates at each sensing node. Figs. 4-5 present the trajectories of the one-step-ahead estimation errors (i.e., $\tilde{x}_{i, k}$ ) of all the sensing nodes. The estimation error variances of all the sensing nodes are proposed in Fig. 67 , which indicate that the proposed algorithm performs quite well.

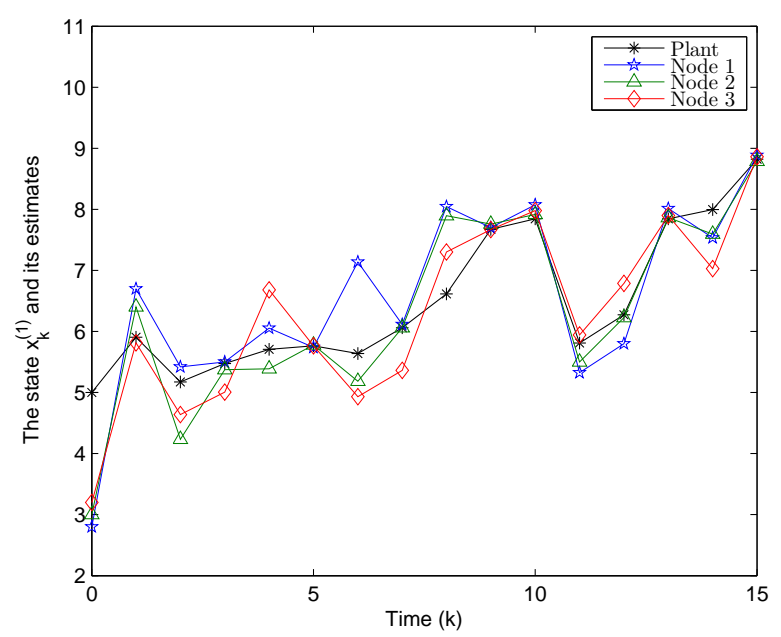

Fig. 2. The state $x_{k}^{(1)}$ and its estimate.

\section{CONCLUSION}

The variance-constrained distributed filtering problem has been studied for a class of discrete time-varying systems with multiplicative noises, unknown but bounded disturbances and deception attacks over sensor networks. A novel deception attack model has been proposed, where the attack signals are injected by the adversary to both control and measurement 


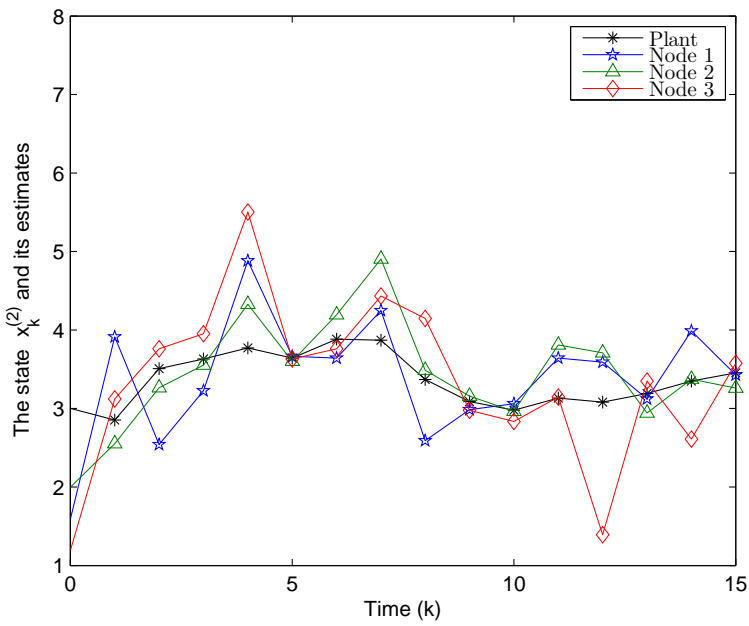

Fig. 3. The state $x_{k}^{(2)}$ and its estimate.

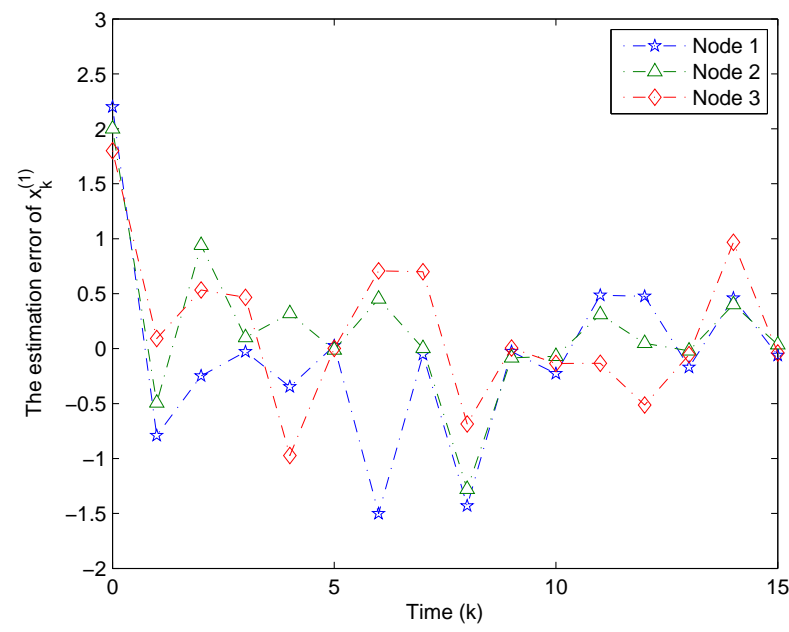

Fig. 4. The estimation error of $x_{k}^{(1)}$.

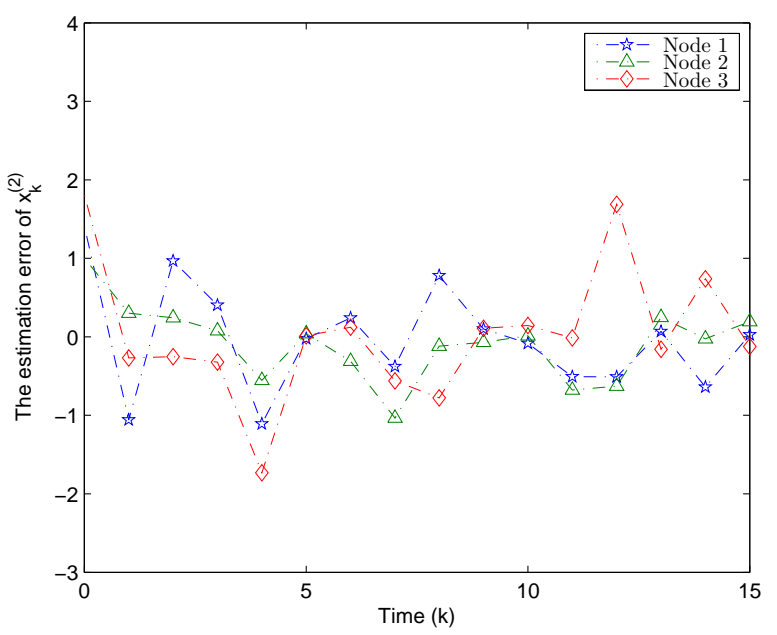

Fig. 5. The estimation error of $x_{k}^{(2)}$. data during the transmission via the communication networks. A sufficient condition has been established for the existence of the required filter satisfying the estimation error variance constraints by means of the RLMI approach. An optimization problem has been presented to seek the filter parameters with the guarantee of the locally minimal estimation error variance at each time instant. Finally, an illustrative example has been used to show the effectiveness and applicability of the proposed algorithm. It is worth mentioning that our proposed filtering scheme is actually a robust technique against deception attacks. In practical engineering, certain attack detectors such as the widely used $\chi^{2}$ detector are usually implemented. However, $\chi^{2}$ detector is only effective to distinguish deception signals obeying Gaussian distribution, which is therefore cannot be applied to prevent the unknown but bounded deception signals considered in this paper. It is of practical significance to exploit novel mechanisms that are capable of detecting other types of attack signals aside from Gaussian noises.

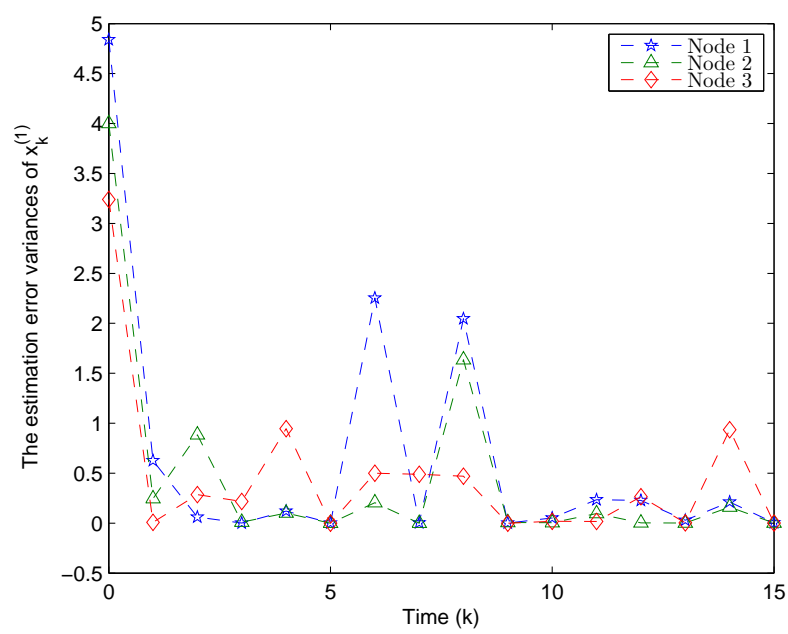

Fig. 6. The variance of $\tilde{x}_{k}^{(1)}$.

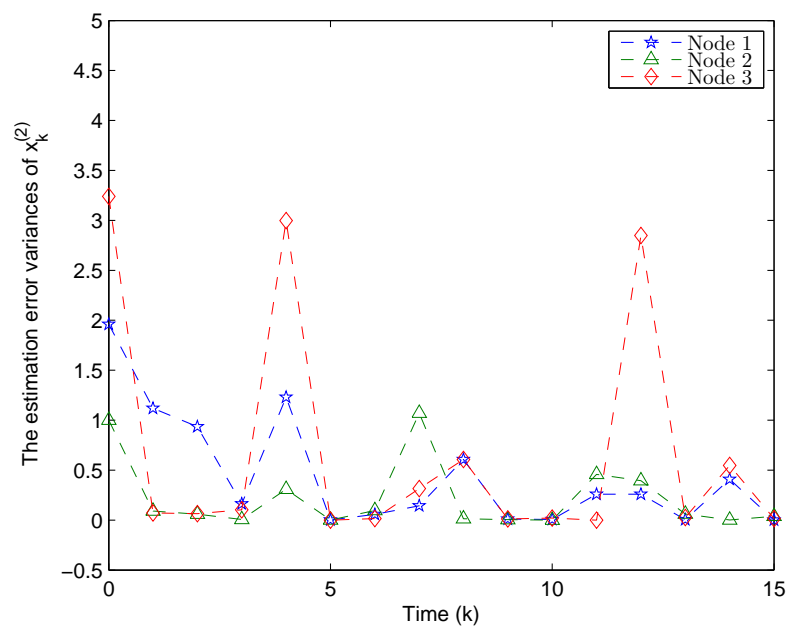

Fig. 7. The variance of $\tilde{x}_{k}^{(2)}$. 


\section{REFERENCES}

[1] M. Blanke, M. Kinnaert, J. Lunze, M. Staroswiecki and J. Schröder, Diagnosis and fault-tolerant control, Springer-Verlag New York, Secaucus, NJ, USA, 2006.

[2] S. Boyd, L. Ghaoui, E. Feron and V. Balakrishnan, Linear matrix inequalities in system and control theory, Philadelphia: SIAM Studies in Applied Mathematics, 1994.

[3] P. Braca, R. Goldhahn, G. Ferri and K. D. LePage, Distributed information fusion in multistatic sensor networks for underwater surveillance, IEEE Sensors Journal, Vol. 16, No. 11, pp. 4003-4014, 2016.

[4] Y. Chen, S. Fei, Y. Li, Stabilization of neutral time-delay systems with actuator saturation via auxiliary time-delay feedback, Automatica, Vol. 52, pp. 242-247, 2015.

[5] D. Ding, Z. Wang and B. Shen, Recent advances on distributed filtering for stochastic systems over sensor networks, International Journal of General Systems, Vol. 43, No. 3-4, pp. 372-386, 2014.

[6] H. Dong, Z. Wang, J. Lam and H. Gao, Distributed filtering in sensor networks with randomly occurring saturations and successive packet dropouts, International Journal of Robust and Nonlinear Control, Vol. 24, No. 12, pp. 1743-1759, 2014

[7] H. Fawzi, P. Tabuada and S. Diggavi, Secure estimation and control for cyber-physical systems under adversarial attacks, IEEE Transactions on Automatic control, Vol. 59, No. 6, pp. 1454-1467, 2014.

[8] Y. K. Foo, Y.C. Soh and M. Moayedi, Linear set-membership state estimation with unknown but bounded disturbances, International Journal of Systems Sciences, Vol. 43, No. 4, pp. 715-730, 2012.

[9] L. El. Ghaoui and G. Calafiore, Robust filtering for discrete-time systems with bounded noise and parametric uncertainty, IEEE Transactions on Automatic Control, Vol. 46, pp. 1084-1089, 2001.

[10] A. Giani, S. Sastry, K. H. Johansson and H. Sandberg, The VIKING project: an initiative on resilient control of power networks, in Proceedings of 2nd International Symposium on Resilient Control Systems, Idaho Falls, ID, pp. 31-35, Aug. 2009.

[11] V. S. Kouikoglou and Y. A. Phillis, Trace bounds on the covariances of continuous-time systems with multiplicative noise, IEEE Transactions on Automatic Control, Vol. 38, No. 1, pp. 138-142, 1993.

[12] A. Kurzhanski and I. Vályi, Ellipsoidal calculus for estimation and control, Boston, MA: Birkhäuser, 1997.

[13] Q. Li, B. Shen, Y. Liu and F. E. Alsaadi, Event-triggered $H_{\infty}$ state estimation for discrete-time stochastic genetic regulatory networks with Markovian jumping parameters and time-varying delays, Neurocomputing, vol. 174, pp. 912-920, 2016.

[14] W. Li, G. Wei, F. Han and Y. Liu, Weighted average consensus-based unscented Kalman filtering, IEEE Transactions on Cybernetics, vol. 46, no. 2, pp. 558-567, 2016

[15] D. Liu, Y. Liu, and F. E. Alsaadi, A new framework for output feedback controller design for a class of discrete-time stochastic nonlinear system with quantization and missing measurement, International Journal of General Systems, vol. 45, no. 5, pp. 517-531, 2016.

[16] Q. Liu, Z. Wang, X. He and D.-H. Zhou, Event-based recursive distributed filtering over wireless sensor networks, IEEE Transactions on Automatic Control, Vol. 60, No. 9, pp. 2470-2475, 2015.

[17] Q. Liu, Z. Wang, X. He and D.-H. Zhou, Event-based $H_{\infty}$ consensus control of multi-agent systems with relative output feedback: the finitehorizon case, IEEE Transactions on Automatic Control, Vol. 60, No. 9, pp. 2553-2558, 2015.

[18] S. Liu, G. Wei, Y. Song and Y. Liu, Extended Kalman filtering for stochastic nonlinear systems with randomly occurring cyber attacks, Neurocomputing, vol. 207, pp. 708-716, 2016.

[19] S. Liu, G. Wei, Y. Song and Y. Liu, Error-constrained reliable tracking control for discrete time-varying systems subject to quantization effects, Neurocomputing, vol. 174, pp. 897-905, 2016.

[20] Y. Liu, W. Liu, M. A. Obaid and I. A. Abbas, Exponential stability of Markovian jumping Cohen-Grossberg neural networks with mixed mode-dependent time-delays, Neurocomputing, Vol. 177, pp. 409-415, 2016.

[21] M. Long, C.-H. Wu and J. Y. Hung, Denial of service attacks on network-based control systems: impact and mitigation, IEEE Transactions on Industrial Informatics, Vol. 1, No. 2, pp. 85-96, 2005.

[22] L. Ma, Z. Wang, H.-K. Lam, F. E. Alsaadi and X. Liu, Robust filtering for a class of nonlinear stochastic systems with probability constraints, Automation and Remote Control, Vol. 77, No. 1, pp. 37-54, 2016.

[23] D. Mascarenas, E. Flynn, C. Farrar, G. Park and M. Todd, A mobile host approach for wireless powering and interrogation of structural health monitoring sensor networks, IEEE Sensors Journal, Vol. 9, No. 12, pp. 1719-1726, 2009.
[24] M. Milanese and A. Vicino, Optimal estimation theory for dynamic systems with set membership uncertainty: an overview, Automatica, Vol. 27, pp. 997-1009, 1991

[25] Y. Mo, S. Weerakkody and B. Sinopoli, Physical authentication of control systems designing watermarked control inputs to detect counterfeit sensor outputs, IEEE Control Systems Magazine, Vol. 35, No. 1, pp. 93 $109,2015$.

[26] R. Olfati-Saber, Distributed Kalman filtering for sensor networks, in Proceedings of 46th IEEE Conference on Decision and Control, Vols. 114, pp. 1814-1820, 2007.

[27] Z.-H. Pang and G.-P. Liu, Design and implementation of secure networked predictive control systems under deception attacks, IEEE Transactions on Control Systems Technology, Vol. 20, No. 5, pp. 13341342, 2012.

[28] B. Sinopoli, C. Sharp, L. Schenato, S. Schaffert and S. S. Sastry, Distributed control applications within sensor networks, Proceedings of the IEEE, Vol. 91, No. 8, pp. 1235-1246, 2003.

[29] A. Teixeira, K. C. Sou, H. Sandberg and K. H. Johansson, Secure control systems: a quantitative risk management approach, IEEE Control Systems Magazine, Vol. 35, No. 1, pp. 24-45, 2015.

[30] J. D. Townsend, M. Saville, S. Hong and R. Martin, Simulator for velocity gate pull-off electronic countermeasure techniques, In: Proc. of 2008 IEEE Radar Conference, Vols. 1-4, pp. 1899-1904, 2008.

[31] V. Ugrinovskii, Distributed robust estimation over randomly switching networks using $H_{\infty}$ consensus, Automatica, Vol. 49, No. 1, pp. 160168,2013

[32] A. Vempaty, O. Ozdemir, K. Agrawal, H. Chen and P. K. Varshney, Localization in wireless sensor networks: Byzantines and mitigation techniques, IEEE Transactions on Signal Processing, Vol. 61, No. 6 , pp. 1495-1508, 2013.

[33] X. Wang and H. Poor, Robust multiuser detection in non-Gaussian channels, IEEE Transactions on Signal Processing, Vol. 47, No. 2, pp. 289-305, 1999.

[34] J. Zhang, R. S. Blum, X. Lu and D. Conus, Asymptotically optimum distributed estimation in the presence of attacks, IEEE Transactions on Signal Processing, Vol. 63, No. 5, pp. 1086-1101, 2015.

[35] J. Zhang, L. Ma and Y. Liu, Passivity analysis for discrete-time neural networks with mixed time-delays and randomly occurring quantization effects, Neurocomputing, Vol. 216, pp. 657-665, 2016.

[36] J. Zhang, L. Ma, M. Lyu, F. E. Alsaadi and Y. Bo, $H_{\infty}$ and $l_{2} / l_{\infty}$ finite-horizon filtering with randomly occurring gain variations and quantization effects, Applied Mathematics and Computation, Vol. 298, pp. 171-187, 2017.

[37] W. Zhang, Z. Wang, Y. Liu, D. Ding and F. E. Alsaadi, Event-based state estimation for a class of complex networks with time-varying delays: a comparison principle approach, Physics Letters A, Vol. 381, No. 1 , pp. $10-18,2017$.

[38] Q. Zhu and T. Başar, Game-theoretic methods for robustness, security, and resilience of cyberphysical control systems: games-in-games principle for optimal cross-layer resilient control systems, IEEE Control Systems Magazine, Vol. 35, No. 1, pp. 46-65, 2015.

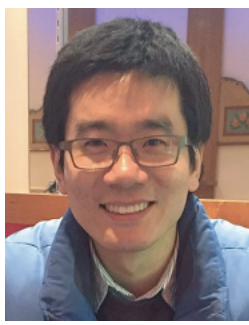

Lifeng Ma received the B.Sc. degree in Automation from Jiangsu University, Zhenjiang, China, in 2004 and the Ph.D. degree in Control Science and Engineering from Nanjing University of Science and Technology, Nanjing, China, in 2010. From August 2008 to February 2009, he was a Visiting Ph.D. Student in the Department of Information Systems and Computing, Brunel University London, U.K. From January 2010 to April 2010 and May 2011 to September 2011, he was a Research Associate in the Department of Mechanical Engineering, the University of Hong Kong.

He is currently an Associate Professor in the School of Automation, Nanjing University of Science and Technology, Nanjing, China, and is currently a Visiting Research Fellow at the King's College London, U.K. His current research interests include nonlinear control and signal processing, variable structure control, distributed control and filtering, time-varying systems and multi-agent systems. He has published more than 20 papers in refereed international journals. He serves as an editor for Neurocomputing. $\mathrm{He}$ is a very active reviewer for many international journals. 


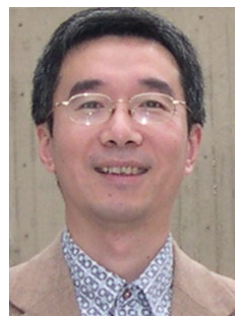

Zidong Wang (SM'03-F'14) was born in Jiangsu, China, in 1966. He received the B.Sc. degree in mathematics in 1986 from Suzhou University, Suzhou, China, and the M.Sc. degree in applied mathematics in 1990 and the Ph.D. degree in electrical engineering in 1994, both from Nanjing University of Science and Technology, Nanjing, China.

$\mathrm{He}$ is currently Professor of Dynamical Systems and Computing in the Department of Information Systems and Computing, Brunel University London, U.K. From 1990 to 2002, he held teaching and research appointments in universities in China, Germany and the UK. Prof. Wang's research interests include dynamical systems, signal processing, bioinformatics, control theory and applications. He has published more than 300 papers in refereed international journals. He is a holder of the Alexander von Humboldt Research Fellowship of Germany, the JSPS Research Fellowship of Japan, William Mong Visiting Research Fellowship of Hong Kong.

Prof. Wang serves (or has served) as the Editor-in-Chief for Neurocomputing and an Associate Editor for 12 international journals, including IEEE TRANSACTIONS ON AUTOMATIC CONTROL, IEEE TRANSACTIONS on CONTROL SySTEMS TECHNOLOGY, IEEE TRANSACTIONS ON NEURAL Networks, IEEE Transactions on Signal Processing, and IEEE TRANSACTIONS ON SYSTEMS, MAN, AND CybERNETICS - PART C. He is a Fellow of the IEEE, a Fellow of the Royal Statistical Society and a member of program committee for many international conferences.

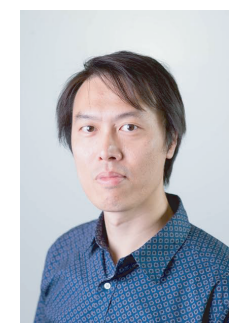

Hak-Keung Lam (M'98-SM'10) received the B.Eng. (Hons.) and Ph.D. degrees from Hong Kong Polytechnic University, Hong Kong, in 1995 and 2000, respectively.

From 2000 to 2005, he was a Post-Doctoral Fellow and Research Fellow with the Department of Electronic and Information Engineering, Hong Kong Polytechnic University. He joined Kings College London, London, U.K., as a Lecturer, in 2005, where he is currently a Reader. He has coedited the books entitled Control of Chaotic Nonlinear Circuits (World Scientific, 2009) and Computational Intelligence and Its Applications (World Scientific, 2012), and coauthored the monograph entitled Stability Analysis of Fuzzy-Model-Based Control Systems (Springer, 2011). His current research interests include intelligent control systems and computational intelligence.

Dr. Lam is an Associate Editor of the IEEE TRAnSACTIONS ON FuZzY Systems, the IEEE TRANSACTIONS ON CIRCUITS AND SySTEMS II: EXPRESS BRIEFS, IET Control Theory and Applications, the International Journal of Fuzzy Systems, and Neorocomputing, and a Guest Editor and an Editorial Board Member for a number of international journals. He served as a Program Committee Member and an International Advisory Board Member for various international conferences, and a Reviewer for various books, international journals, and international conferences.

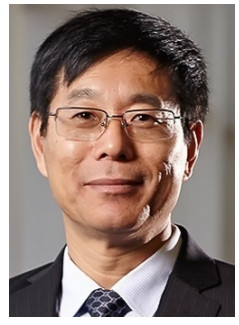

Qing-Long Han (M'09-SM'13) received the B.Sc. degree in mathematics from Shandong Normal University, Jinan, China, in 1983, and the M.Sc. and $\mathrm{Ph} . \mathrm{D}$. degrees in Control Engineering and Electrical Engineering from East China University of Science and Technology, Shanghai, China, in 1992 and 1997. respectively.

From September 1997 to December 1998, he was a Post-doctoral Researcher Fellow with the Laboratoire d'Automatique et d'Informatique Industielle (now renamed as Laboratoire d'Informatique et d'Automatique pour les Systémes), Ecole Sup érieure d'Ing'enieurs de Poitiers (now renamed as Ecole Nationale Supérieure d'Ingé nieurs de Poitiers), Universit é de Poitiers, France. From January 1999 to August 2001, he was a Research Assistant Professor with the Department of Mechanical and Industrial Engineering at Southern Illinois University at Edwardsville, USA. From September 2001 to December 2014, he was Laureate Professor, Associate Dean (Research and Innovation) with the Higher Education Division, and Founding Director of the Centre for Intelligent and Networked Systems at Central Queensland University, Australia. From December 2014 to May 2016, he was Deputy Dean (Research), with the Griffith Sciences, and Professor with the Griffith School of Engineering, Griffith University, Australia. In May 2016, he joined Swinburne University of Technology, Australia, where he is currently Pro Vice-Chancellor (Research Quality) and Distinguished Professor His research interests include networked control systems, neural networks, time-delay systems, multiagent systems, and complex systems.

Professor Han was appointed Chang Jiang (Yangtze River) Scholar Chair Professor by the Ministry of Education, China, in March 2010. He is one of The World's Most Influential Scientific Minds: 2014, The World's Most Influential Scientific Minds: 2015, and The World's Most Influential Scientific Minds: 2016. He is a Highly Cited Researcher in Engineering according 1153 to Thomson Reuters. 